A si mol e opt i cal method for measuring the vi brat i on ampl i tude of a speaker

\begin{tabular}{|l|l|}
\hline 著者 & $\begin{array}{l}\text { UEDA Nasahi r o, YANAGUCH Toshi hi ko, KAK UCH } \\
\text { H r oki , SUGA H r oshi }\end{array}$ \\
\hline $\begin{array}{l}\text { j our nal or } \\
\text { publ i cat i on t i t l e }\end{array}$ & Opt i cs and Laser s i n Engi neeri ng \\
\hline vol une & 32 \\
\hline number & 1 \\
\hline page r ange & $21-28$ \\
\hline year & $1999-07$ \\
\hline URL & ht t p: //hdl . handl e. net /10098/1467 \\
\hline
\end{tabular}




\title{
A simple optical method for measuring the vibration amplitude of a speaker
}

\author{
Masahiro Ueda ${ }^{\mathrm{a}}$, Toshihiko Yamaguchi ${ }^{\mathrm{a}}$, Hiroki Kakiuchi ${ }^{\mathrm{a}}$, \\ Hiroshi Suga ${ }^{\mathrm{b}}$
}

\author{
${ }^{a}$ Faculty of Education and Regional Studies, Fukui University, 3-9-1 Bunkyo, Fukui 910-0017, Japan \\ ${ }^{\mathrm{b}}$ Faculty of Information Science, Osaka Institute of Technology, 1-79-1 Kitayama, Hirakata, \\ Osaka 573-0171, Japan
}

\begin{abstract}
A simple optical method has been proposed for measuring the vibration amplitude of a speaker vibrating with a frequency of approximately $10 \mathrm{kHz}$. The method is based on a multiple reflection between a vibrating speaker plane and a mirror parallel to that speaker plane. The multiple reflection can magnify a dispersion of the laser beam caused by the vibration, and easily make a measurement of the amplitude. The measuring sensitivity ranges between sub-microns and $1 \mathrm{~mm}$. A preliminary experiment verified the reliability of this method.
\end{abstract}

\section{Introduction}

A sound speaker for a high frequency of approximately $10 \mathrm{kHz}$ is usually composed of thin mirror-like metal plates. The efficiency of the speaker will be determined by the material, thickness, and tension of the metal. In practice, however, efficiency is determined by measuring the vibration amplitude due to an electric input power. Therefore, in order to evaluate the speaker quality, it is important and urgent to develop a practical method to measure the vibration of the micro-speaker with radius about $5 \mathrm{~mm}$.

Generally, a vibration with amplitude above a few tens microns are measured by using a mechanical method [1]. Optical interferometry, in particular optical holography [2], has been used to measure vibration with amplitude between $0.01 \mu \mathrm{m}$ and several microns. As to a vibration with amplitude about $10 \mu \mathrm{m}$ as that of our speaker, 
Doppler method is probably the only way to measure it. However, it is, in itself, a method to measure a velocity, and a particular improvement in optical arrangement and electronic circuit is necessary to use it as a detector for the vibration amplitude [3]. The system, therefore, is not simple and cost-effective for our case.

In this paper, a simple method through measuring the multi-reflected laser beam "dispersion" due to vibration to measure vibration amplitude above $10 \mu \mathrm{m}$ is proposed.

\section{Principle and method}

The basic idea of the proposed method is to use a plane mirror just on a vibrating speaker and to cast a multi-reflected laser beam between them on the detectors such as a screen and a CCD camera. The laser light reflected on the vibrating mirror disperses and becomes obscure slightly at the edge of the laser beam. Actually, the reflected laser beam is not dispersed but is deflected slightly from an original direction due to the vibration, but it seems dispersed, as the vibration frequency is so high as to detect it with eye and detector. We call the phenomenon "a dispersion" in this study. The dispersion increases linearly, according to the vibration amplitude; that is, the vibration amplitude can be detected by means of the dispersion. The dispersion is, however, usually very small. The multiple reflection increases the dispersion; that is, multiple reflection leads to high sensitivity of measurement.

A thin plate of the speaker will, actually, vibrate in such a fashion as circular arc shown in Fig. 1. However, we decompose the circular arc into two linear planes; i.e., an inclined plane $P_{1}$ and a horizontal plane $P_{2}$, for a convenience of theoretical analysis. That is, an actual dispersion can be approximated by a combination of the dispersions on planes $P_{1}$ and $P_{2}$.

\subsection{Laser beam dispersion on the inclined plane $P_{1}$}

Fig. 2 shows a comparison of the laser beam reflections between parallel plates and non-parallel plates. The laser beam reflected between non-parallel plates deflects from

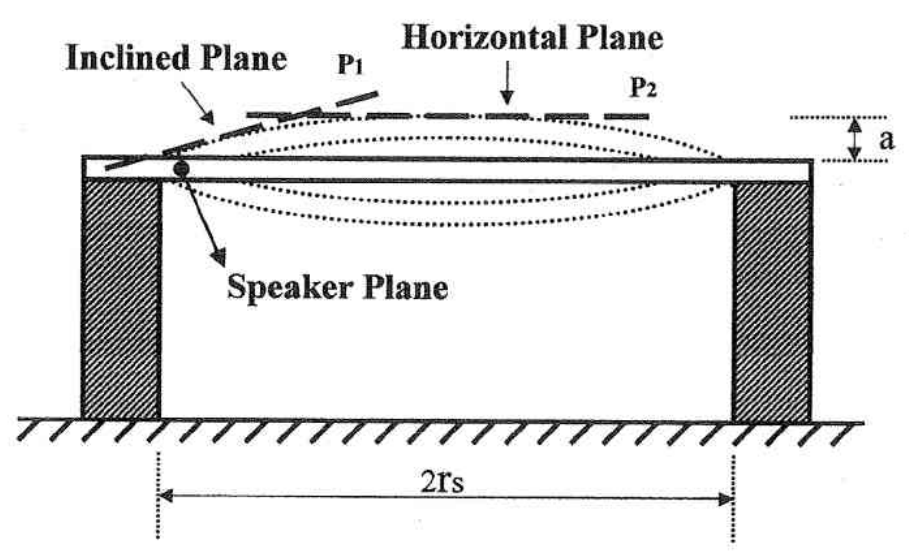

Fig. 1. Decomposition of practical vibration plane into two flat planes; i.e., an inclined plane and a horizontal plane. 


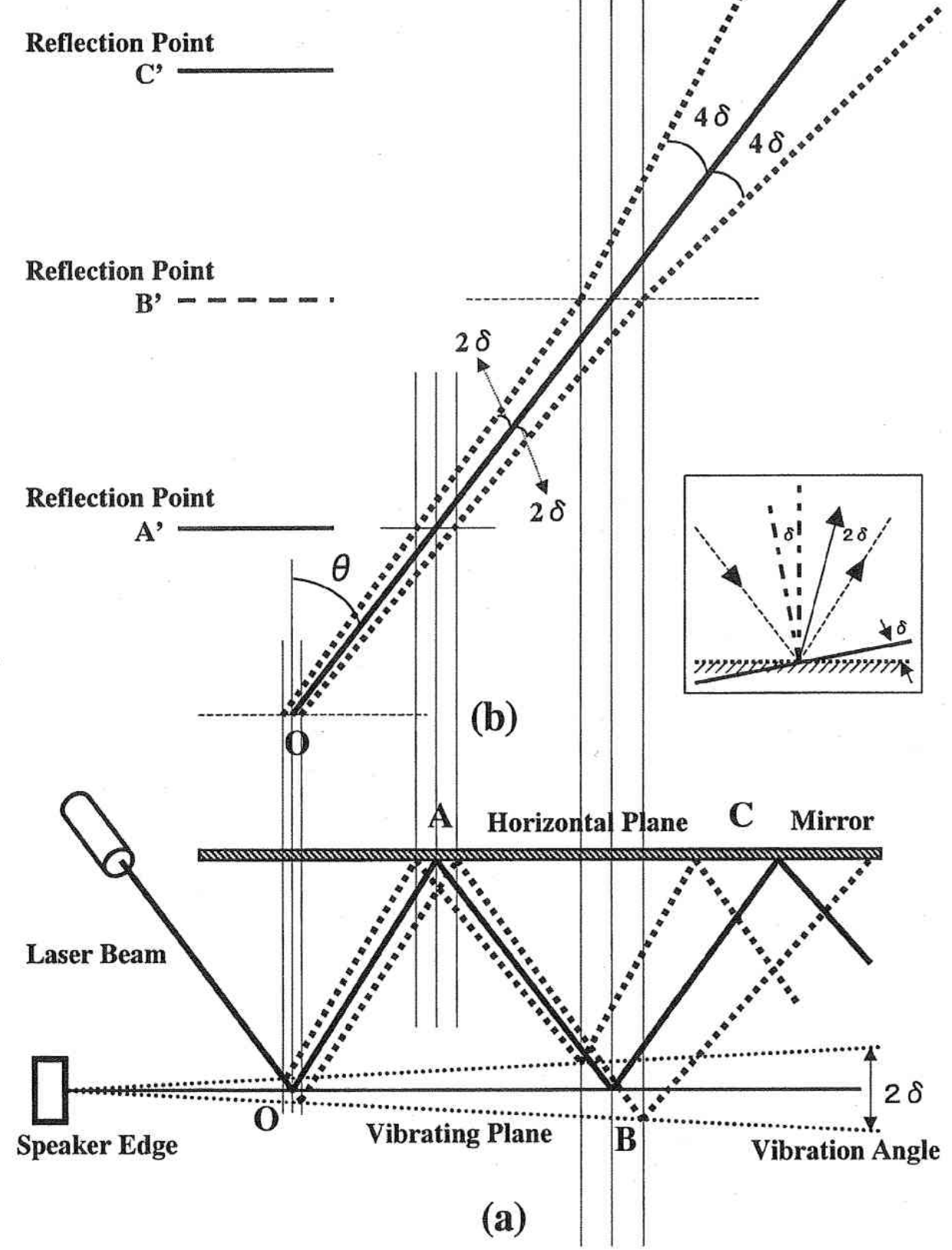

Fig. 2. Multiple reflections between parallel mirrors and non-parallel mirrors: (a) practical multiple reflection; (b) laser beam profile symmetrical to a plane mirror.

the one reflected between parallel plate. As was shown in a cut, when the mirror inclines slightly, $\delta$, the reflected light deviates $2 \delta$ from the original direction and this causes a dispersion of the laser beam. The mean inclination, $\langle\delta\rangle$, can be approximated by

$$
\langle\delta\rangle=a / r_{\mathrm{s}},
$$

where $a$ is vibration amplitude at the central region and $r_{\mathrm{s}}$ is a radius of the speaker. The light deviates, finally, $2 n\langle\delta\rangle$ after $n$ times multi-reflection at a vibrating plane, as shown in Fig. 2(b). The dimension of the dispersion on the screen due to the vibration, $W$, will, then, be

$$
W=2 n\langle\delta\rangle \times R=2 n a R / r_{\mathrm{s}},
$$


at a distance $R$ from the vibration plane to the screen. Thus, the multiple reflection used in this method acts as an amplifier of the beam dispersion caused by the vibration amplitude. The amplification, i.e., the efficacy of multiple reflection, which is defined by $W / W_{0}$, can be given by

$$
W / W_{0}=n,
$$

where $W_{0}$ is the beam spread represented by a single reflection, $n=1$. For example, we can obtain $W=2 \mathrm{~mm}$ for a practical dimension of $a=10 \mu \mathrm{m}, r_{\mathrm{s}}=5 \mathrm{~mm}$, $R=100 \mathrm{~mm}$, and $n=5$. This value of $W=2 \mathrm{~mm}$ can easily be observed by the naked eye. The actual inclination will be larger than the mean value given by (1) since the central zone of the speaker plane becomes horizontal. Thus, the actual spread dimension will be larger than $W$. This causes an error and will be discussed in Section 3 .

\subsection{Laser beam dispersion on the horizontal plane $P_{2}$}

In this case, in contrast to the case of the inclined plane, the laser beam does not diverge in itself in a free space since the direction of the laser beam does not deviate from that on the stationary plane. This renders the amplitude measurement difficult. However, application of multiple reflection can overcome this difficulty.

Fig. 3 shows an illustration of the multiple reflection between the horizontally vibrating plane and the plane mirror. The reflection on the horizontally vibrating plane does not deflect the direction of the laser beam, but it rather increases the spread as shown in Fig. 3(a). The spread of the laser beam at the vibrating plane, $W^{\prime}$, can be expressed as

$$
W^{\prime}=4 a \sin \theta .
$$

Thus, no spread exceeds $4 a$. The spread after a multiple reflection, however, can be larger than $4 a$. The spread after an $n$ times reflections can be calculated from a simple geometric consideration, as follows:

$$
W=2 a L \cos \theta / d,
$$

where $L$ is an effective length of the vibrating plane mirror, $\theta$ is an incident angle of the laser beam, and $d$ is a distance between the vibrating plane and the mirror. The amplification of the spread due to multiple reflection defined by $W / W^{\prime}$ is then

$$
W / W^{\prime}=L /(2 d \times \tan \theta) .
$$

High amplification can, therefore, be obtained by using a large $L$, a small $d$, and a small $\theta$. Remarkably small values for $d$ would be impractical. We choose $d=0.5 \mathrm{~mm}, L=2 \mathrm{~mm}$, and $\theta=\pi / 18$, for $a=10 \mu \mathrm{m}$ with a practical speaker dimension of $r_{\mathrm{s}}=5 \mathrm{~mm}$. We then obtained $W / W^{\prime}=11.34$, and $W=78.8 \mu \mathrm{m}$, which may be measured easily by CCD camera.

Practical beam spread is a superposition of both the spreads expressed by (2) and (5),

$$
W_{\mathrm{p}}=2 n a R / r_{\mathrm{s}}+2 a L \cos \theta / d .
$$


(a)

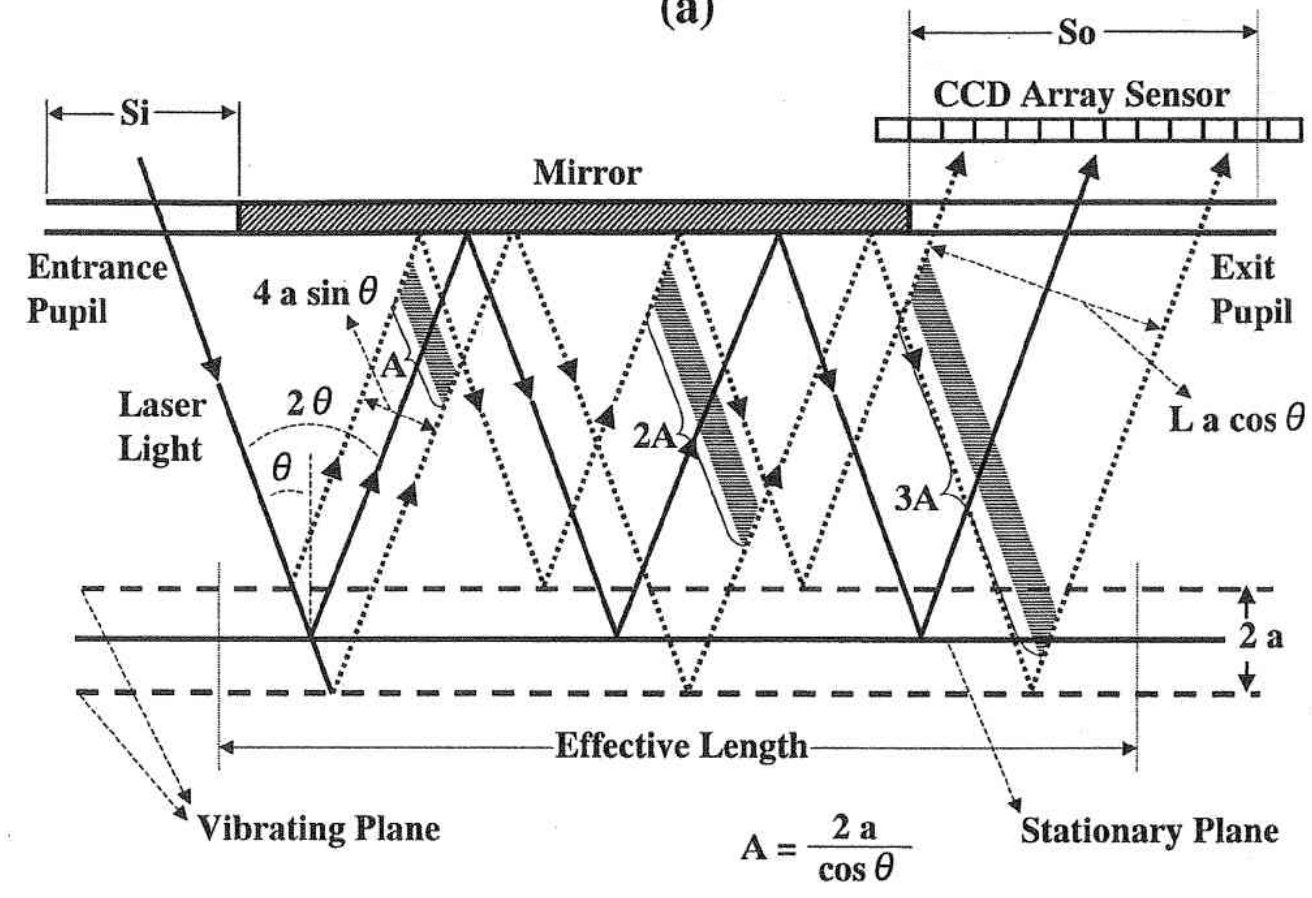

(b)
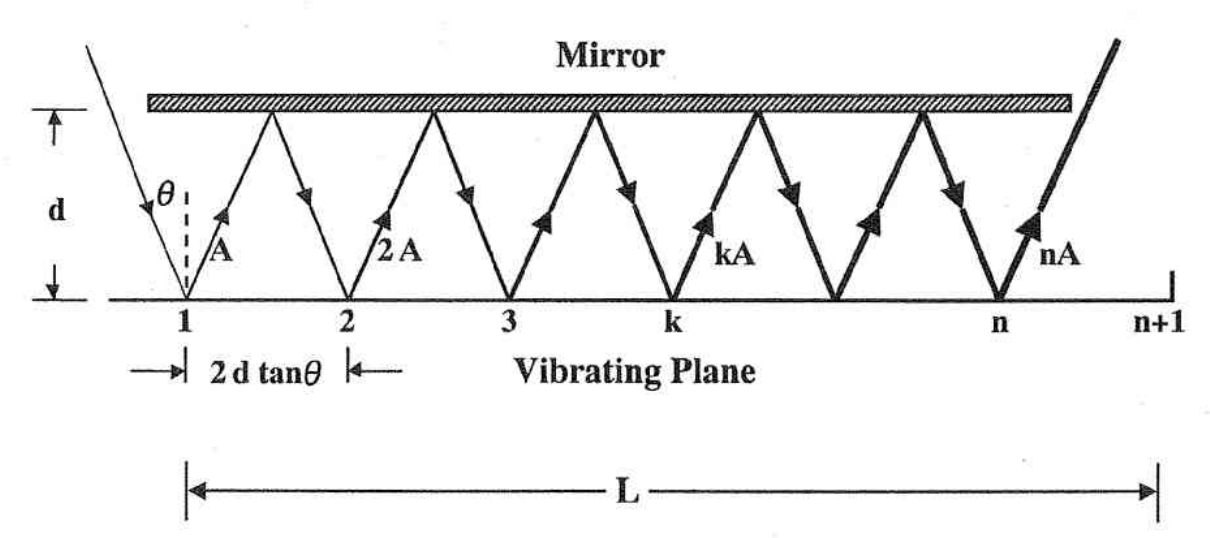

Fig. 3. Multiple reflection between a horizontally vibrating plane and a stationary mirror: (a) illustration of the beam spread; (b) aspect of multiple reflection.

As discussed above, the dispersion on the inclined plane is rather large as compared with that on the horizontal plane for the speaker used in this study (dimension of $r_{\mathrm{s}}=5 \mathrm{~mm}$ and mean vibration amplitude of $\left.a=10 \mu \mathrm{m}\right)$.

\section{Experimental results and discussions}

A preliminary experiment was conducted by using a large-scaled optical arrangement and by replacing the vibration amplitude with the static displacement of the mirror. That is, the displacement was considerably enlarged as compared with the practical dimensions for a convenience of measurement. 
Fig. 4 shows an example of a laser beam deflection on the inclined plane mirror. The divergence of the laser beam increased linearly in accordance with the multiple reflection as predicted in Section 2.1. Table 1 shows a laser beam spread on the horizontal plane mirror for some values of $d$ and $\theta$. The spread, $W / W^{\prime}$, also agreed well with the calculated values given by (6). From these results, the proposed method in this paper was found to be valid.

In this preliminary experiment, the laser beam from a $\mathrm{He}-\mathrm{Ne}$ laser was used directly, but a laser beam with a small cross-section would be desirable in a practical use since a speaker plane is very small. This can be done easily by using the mirror with a small entrance pupil as shown in Fig. 3(a). However, too small a pupil would cause a diffraction effect, which makes a detection of the beam spread difficult, and a proper beam size will be $500-1000 \mu \mathrm{m}$.

Fig. 5 shows an illustrative intensity profile of the laser beam on the observation plane. The broken curve represents the cross-sectional intensity of the laser beam itself, which is obtained by multiple reflection on a stationary mirror, and the solid curve represents the intensity profile obtained by multiple reflection on a vibrating plane. The intensity will, thus, fade around the edge region, $W_{\mathrm{p}}$, due to the vibration, as discussed in Sections 2.1 and 2.2. The value can be detected by using a CCD array sensor and a microscopic observation.

In this preliminary experiment, the beam spread due to both inclined planes and parallel planes was detected separately. However, these spread are incorporated and cannot be detected separately in practice, as shown in (7). This causes a measuring error of this method. The considerable error factors of this method will result from a diffraction effect due to too small an entrance pupil, a laser beam divergence and a detection error. The first and the second factors will be eliminated since only a beam spread, $W_{\mathrm{p}}$, due to vibration can be detected separately. Therefore, the remaining error factors will be the detection errors arising from a resolution of the detector and an indistinctness of the causes of the beam spread. The later error cannot be discussed theoretically; it can, only, be discussed experimentally. Only the former error factor

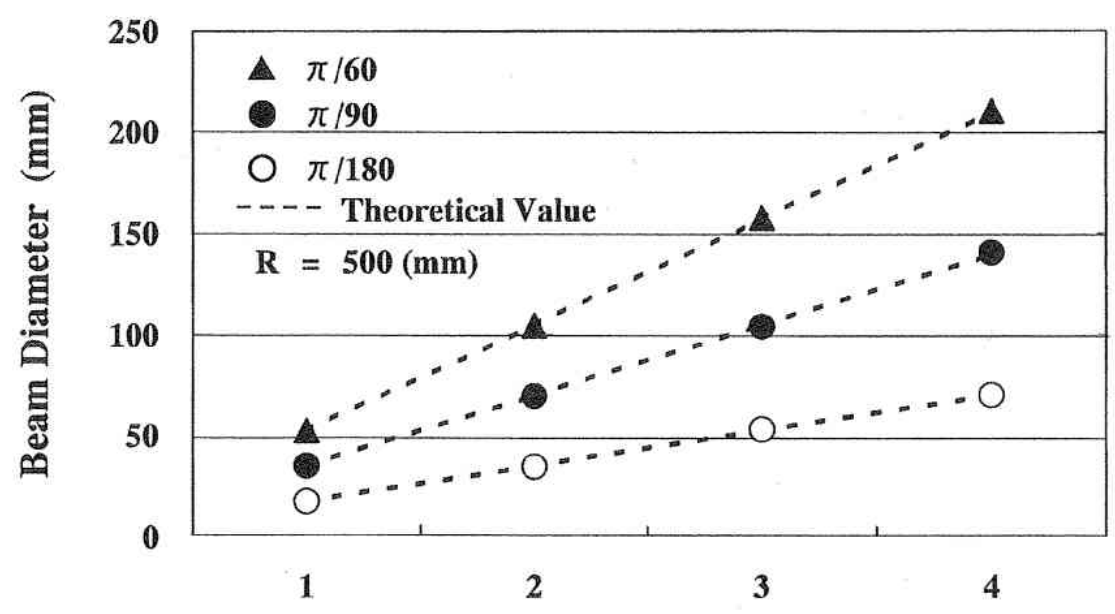

Reflection Times n

Fig. 4. The laser beam deflection on the inclined plane mirror. 
Table 1

The laser beam spread on the horizontal plane mirror under the experimental conditions of $a=1 \mathrm{~mm}$ and $L=100 \mathrm{~mm}$

\begin{tabular}{lllll}
\hline$\theta$ & $d(\mathrm{~mm})$ & $W^{\prime}(\mathrm{mm})$ & $W(\mathrm{~mm})$ & \multicolumn{2}{c}{$W / W^{\prime}$} \\
\hline$\pi / 4$ & \multirow{2}{*}{10} & Cal. 2.8 & Cal. 14.1 & Cal. 5.0 \\
& & Exp. 2.9 & Exp. 14.1 & Exp. 4.9 \\
& 20 & Cal. 2.8 & Cal. 7.0 & Cal. 2.5 \\
& & Exp. 2.8 & Exp. 7.2 & Exp. 2.6 \\
$\pi / 3$ & & & Cal. 10.0 & Cal. 2.9 \\
& \multirow{2}{*}{10} & Cal. 3.5 & Exp. 9.8 & Exp. 2.8 \\
& \multirow{2}{*}{20} & Exp. 3.5 & Cal. 5.0 & Cal. 1.4 \\
& & Cal. 3.5 & Exp. 4.9 & Exp. 1.4 \\
\hline
\end{tabular}

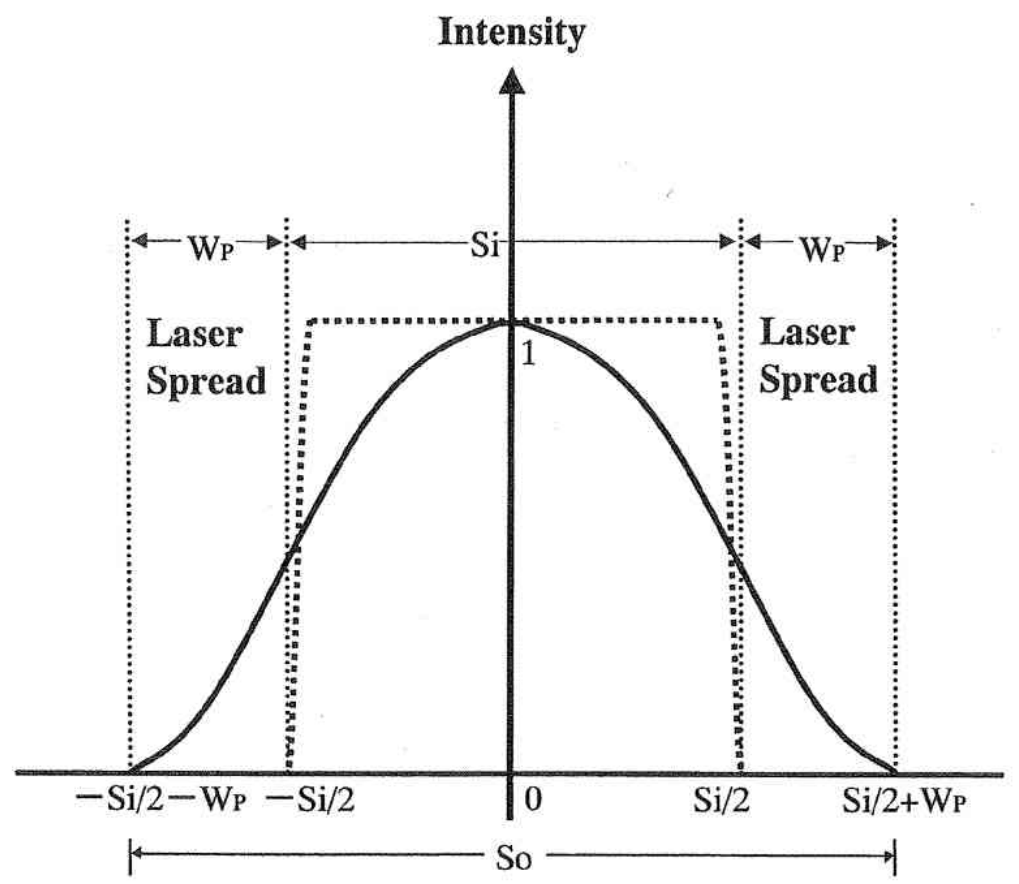

Fig. 5. An illustration of a light intensity profile on an observation plane. Broken curve represents the intensity reflected on the stationary plane, and the solid curve represents that on the vibrating plane.

will, then, be discussed in this paper. We can obtain the measuring errors for the vibration amplitude, $\Delta a$, from (2) and (5), as follows:

$$
\Delta a=r_{\mathrm{s}} /(2 n R) \times \Delta W \quad \text { and } \quad \Delta a=\{d /(2 L \cos \theta)\} \times \Delta W,
$$

where $\Delta W$ is a resolution of the detector. The measuring error on the inclined plane mirror is, then, $\Delta a=(1 / 200) \times \Delta W$ for the previous values of $r_{\mathrm{s}}=5 \mathrm{~mm}, n=5$ and $R=100 \mathrm{~mm}$, and that on the parallel mirror is $\Delta a=(0.127) \times \Delta W$ for the previous values of $d=0.5 \mathrm{~mm}, L=2 \mathrm{~mm}$ and $\theta=\pi / 18$. The resolution of the CCD camera is usually a few microns and then the measuring error due to a resolution of the detector can be sub-microns. 


\section{Conclusion}

An optical method for measuring vibration amplitude of a speaker has been proposed and verified experimentally. The method, which is very simple, uses multiple reflection between a vibrating plane and a plane mirror parallel to that vibrating plane. The measuring sensitivity of this method ranges between sub-microns and $1 \mathrm{~mm}$, which is sufficiently high for practical use.

\section{References}

[1] Den Hartog JP. Mechanical vibrations, 4th ed. New York: McGraw-Hill, 1956. [Taniguchi O, Fujii S, Trans., Corona, 1998, p. 63.]

[2] Collier RJ, Burckhardt CB, Lawrence HL. Optical holography. New York: Academic Press, 1971. p. 438-41.

[3] Takahashi K, et al., editors. Handbook for the sensors. Tokyo: Asakura, 1991, p. 324. 